\title{
Transtorno Dismorfico Corporal (Tdc) En Pacientes De Cirugía Estética.
}

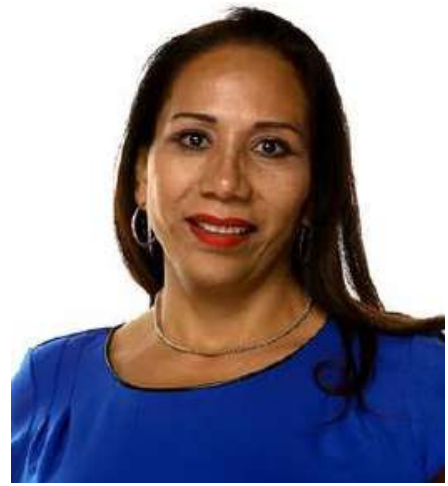

Dra. María Teresa Zambrana Rojas

Hospital San Vicente de Paul

Cochabamba - Bolivia

\section{"Body Dysmorphic Disorder in cosmetic surgery patients"}

\section{RESUMEN}

El trastorno dismórfico corporal (TDC) (antes - dismorfofobia) es un trastorno somatomorfo que consiste en una preocupación fuera de lo normal por algún defecto, ya sea real o imaginado, percibido en las características físicas propias, es una trastorno psiquiátrico con presentación de hasta un $20 \%$ de los pacientes que solicitan cirugía estética.

Debido a sus diversas presentaciones, TDC debe ser diagnosticado, buscado y reconocido por el cirujano plástico de lo contrario, el cirujano tendrá invariablemente que tratar con un paciente profundamente insatisfecho.

Los pacientes con TDC odian partes o todo su cuerpo y puede buscar la cirugía estética como una solución en reiteradas oportunidades.

El diagnóstico, reconocimiento y aplazamiento de la cirugía para los pacientes con TDC es lo que se recomienda sobre todo porque los estudios han demostrado la propensión de estos pacientes a denunciar, a litigar, amenazar e incluso perjudicar o matar a su cirujano.

El objetivo del trabajo es describir las características del TDC en el contexto de la consulta de Cirugía Estética, considerando su prevalencia, comorbilidades y las consecuencias de los procedimientos quirúrgicos en la salud mental de este tipo de pacientes. Considerando que es fundamental la detección del TDC en la consulta de Cirugía Estética para una derivación oportuna de estos pacientes hacia un tratamiento de estos pacientes.

\section{PALABRAS CLAVE}

Trastorno dismórfico corporal - Salud mental - Cirugía Estética - Selección de pacientes.

\section{ABSTRACT}

Body dysmorphic disorder (DBS) (previously known as dysmorphobia) is a somatomorphic disorder that consists of an outof-normal concern for some defect, whether real or imagined, perceived in one's own physical characteristics.Is a psychiatric illness experienced by up to $20 \%$ of patients requesting aesthetic surgery.

Because of its diverse presentations, should be searched out and recognized by the plastic surgeon Otherwise, the unwary surgeon will invariably have to deal with a profoundly dissatisfied patient. Patients with DBS hate some part of their bodies and may seek out cosmetic surgeries a solution. However, they invariably are not satisfied despite the objective result. These patients need be diagnosed, psychological counseling and referral to psychiatrists with a special interest in body image. Recognition and deferral of surgery for DBS patients is advised because findings have shown the propensity of these patients to denounce, litigate, threaten, and even harm or kill their surgeon. The objective of this study is to describe the characteristics of BDD in the context of the Aesthetic or Cosmetic Surgery consultation, considering its prevalence, comorbidities and the consequences of surgical procedures on the mental health of these patients. Considering that it is fundamental the detection of DBS in the Cosmetic Surgery consultation for a timely referral of these patients towards a treatment of these patients. 


\section{KEYWORDS:}

Body dysmorphic disorder - Mental health - Psychological outcome Aesthetic surgery - Patient selection.

\section{INTRODUCCIÓN}

Los pacientes con trastorno dismórfico corporal viven angustiados, tratando de ocultar su "grave defecto". Muchos de ellos creen que la única solución a su problema es la cirugía plástica.

Los cirujanos plásticos que se encuentran continuamente con pacientes que recurren a la cirugía plástica como solución a problemas psicológicos, que no lograran ser solucionados a través de una cirugía, generando conflictos por parte del paciente por las expectativas inalcanzables no cumplidas.

Este trastorno es muy diferente de la vanidad o el narcisismo. Los pacientes con TDC tienen miedo, están muy acomplejados. Se avergüenzan de sí mismos, en ellos las tasas de depresión y suicidio son altas. El trastorno se encuentra con igual frecuencia en hombres y en mujeres. (Alrededor de la mitad de los pacientes con TDC no son conscientes de que sufren un trastorno psicológico, mientras que los demás saben que objetivamente su físico es normal.2

\section{HISTORIA}

Descrito por primera vez en 1891 por el italiano Enrico Morselli, con el término dismorfofobia El Trastorno Dismórfico Corporal (TDC) es una entidad psiquiátrica.1.

En 1987 la Asociación Psiquiátrica Americana incorporó esta entidad en el DSM-III-R. Desde entonces el término dismorfofobia en desuso, ha sido reemplazado por el de Trastorno Dismórfico Corporal (5). Los criterios diagnósticos de la última versión del DSM-IV-TR (3) destaca la excesiva preocupación de los pacientes por algún defecto imaginario de su aspecto físico.

Las personas que sufren TDC suelen intentar corregirlas las anomalías que perciben ocultándolas de maneras ingeniosas y en muchos casos recurriendo a la Cirugía Estética. Este fenómeno ha producido una extensa discusión entre cirujanos plásticos que sostienen que los pacientes con TDC no se verían beneficiados por los procedimientos

En 1997, el Manual de Diagnóstico y Estadística de Mental Disorders, $4^{\text {a }}$ edición (DMS-4), publicado por La Asociación Psiquiátrica Americana, clasifica el TDC como un trastorno somatomorfo. LoS hallazgos muestran que TDC está en aumento en la población general, con un Incidencia del 1-2 \% de la población mundial reúnen los criterios diagnósticos propios del TDC.9

\section{ETIOLOGÍA}

El TDC suele comenzar en la adolescencia e ir disminuyendo con la edad, aunque en ocasiones puede persistir en la edad adulta, es mucho más común entre jóvenes de clase alta, con pocos o ningún defecto físico, influenciados por los medios de comunicación, redes sociales y los altos estándares de belleza actual, con una percepción errónea o exagerada de defectos físicos imperceptibles o imaginarios, afecta a hombres y mujeres por igual. La investigación muestra diferentes factores que pueden estar involucrados, que pueden estar combinados, puede estar asociada con trastornos alimenticios, como el síndrome del comedor compulsivo, anorexia nerviosa o la bulimia, la fobia social o ansiedad social.2

\section{CARACTERÍSTICAS DEL PACIENTE CON TDC}

Las causas del TDC difieren de una persona a otra. Sin embargo, la mayoría de los investigadores creen que podría ser una combinación de factores biológicos, psicológicos y ambientales de su pasado o presente. Malos tratos, abuso o abandono pueden ser también factores contribuyentes.

El inicio de los síntomas ocurre con frecuencia en la adolescencia, donde comienzan la mayoría de críticas personales relacionadas con la imagen corporal, aunque los casos de aparición de TDC en niños y adultos mayores también esta descrita.1

El trastorno provoca deterioro en la calidad de vida; suele presentarse con trastorno depresivo mayor y la fobia social, con ideación suicida de alrededor del $80 \%$, casos extremos de TDC puede ser considerados factores de riesgo para el suicidio.

El TDC generalmente de curso crónico con síntomas que tienden a persistir o empeorar con el tiempo si no se tratan por muchos años antes de decidirse a buscar ayuda psicológica o psiquiátrica.

\section{FACTORES DE RIESGO}

Se han descrito la existencia de varios factores de riesgo para TDC:

- Trastorno obsesivo-compulsivo

- Predisposición genética.

- Rasgos de personalidad tales como perfeccionismo, introversión, timidez y temperamento ansioso.

- Traumas en la infancia causados por constantes burlas, insultos, motes y humillación referido al aspecto físico.

- Bullying o cualquier otra forma de maltrato psicológico.

- Aislamiento y rechazo social.

- Malas relaciones interpersonales.

- Problemas psicológicos o emocionales tempranos.

- Influencias paterno-filiales: padres sobreprotectores o exigentes; maltrato psicológico; abandono; separación; carencias afectivas o emocionales.

- Historia crónica de problemas dermatológicos o estigmas físicos o complejos de la adolescencia (como por ejemplo: el acné), como así también el someterse a cirugías estéticas varias. 
- Sensibilidad estética y sobrevaluación de la imagen o apariencia.5

\section{RELACIÓN CON LA CIRUGÍA ESTÉTICA}

La preocupación de los cirujanos plásticos por el TDC está completamente justificada porque existe una mayor prevalencia del trastorno en sus consultas y la sub detección de estos pacientes porque pasa fácilmente desapercibido, a la hora del diagnóstic0.4

La importancia de un diagnóstico permitirá al cirujano a excluir estos pacientes de cirugías que pretendan corregir defectos imaginarios y derivar a un tratamiento adecuado los casos de TDC y sus posibles comorbilidades psiquiátricas.

Todo paciente en el que se sospeche TDC debe ser derivado a un psiquiatra, siendo el tratamiento de elección psicoterapia y farmacoterapia 4.

El estrés que significa para estos pacientes el no ver una mejoría tras someterse a una cirugía puede empeorar la condición. La agresión verbal, física e incluso las acciones judiciales pueden evitarse de esta forma si logramos excluir los casos de afectación grave 5 .

\section{DIAGNÓSTICO}

El cirujano plástico debe estar consciente y saber identificar a un paciente con trastorno dismórfico corporal (TDC) o la posibilidad de la presencia de este, y así remitir al paciente a un profesional en salud mental. Los pacientes con trastorno dismórfico corporal (TDC) son extremadamente despectivos con respecto a su apariencia física, excediendo los límites de una evaluación objetiva, la obsesión por la apariencia física conduce a que el paciente se aísle de la sociedad, y que pase la mayor parte del tiempo con dicha preocupación, lo que de manera eventual va a causar interferencia con su vida profesional y personal.5.

Ciertos rasgos de personalidad en este tipo de pacientes permiten hacer el diagnóstico y estos pueden variar de un individuo a otro, como la inseguridad emocional, sensibilidad, introversión, timidez, inhibición, necesidad imperiosa de recibir la aprobación de los demás, especial sensibilidad al rechazo y a la crítica, vulnerabilidad, excesiva autoconsciencia, obsesividad, ansiedad, narcisismo, hipocondría, neuroticismo, perfeccionismo, autoestima, autoimagen y auto concepto pobre, dificultad para las relaciones interpersonales, déficit en habilidades sociales y asertividad, rasgos de personalidad por evitación, personalidad dependiente, personalidad obsesivo compulsiva (Cluster C), rasgos de personalidad limítrofe e histriónica (Cluster B).

Las zonas del cuerpo que con mayor frecuencia se reportan como causa de preocupación en el paciente con trastorno dismórfico corporal (TDC) incluyen: la piel (73\%), cabello (56\%), peso (55\%), nariz ( $37 \%)$, dedos del pie $(36 \%)$ abdomen ( $22 \%)$, senos $(21 \%)$, ojos (20\%), muslos (20\%), dientes (20\%), piernas $(18 \%)$, rasgos faciales en general (14\%), tamaño y forma de la cara $(12 \%)$, labios $(12 \%)$, glúteos $(12 \%)$,barbilla $(11 \%)$, orejas $(9 \%)$, cintura $(9 \%)$, genitales $(8 \%)$, frente $(6 \%)$, pies $(6 \%)$, manos $(6 \%)$, la mayoría de las personas con trastorno dismórfico corporal (TDC) tienen más de un área corporal motivo de preocupación. 6

Aproximadamente 6 al $15 \%$ de los pacientes que se presentan con el cirujano plástico en busca de una cirugía, pueden padecer TDC. La adicción que presentan ciertos pacientes a la cirugía estética es un signo de alarma; se ha determinado que los hombres que se han sometido a una rinoplastia son tres veces más susceptibles que las mujeres a estar inconformes con los resultados del procedimiento quirúrgico. 6 El paciente busca la aprobación social, mejorar sus relaciones personales, aumentar el número de seguidores en redes sociales, tener un mayor éxito en el empleo, un paciente que no se encuentra satisfecho puede considerar que una cirugía fallida es causal de que no se cumplan las expectativas planeadas al pensar realizarse un procedimiento estético y la solución a su problema resulta en someterse a otro procedimiento quirúrgico estético, que cambiará sus vidas y es probable que se sienta decepcionado cuando los cambios físicos no conducen al resultado esperado previsto.7

\section{TRATAMIENTO}

La sospecha de trastorno dismórfico corporal (TDC) es indicativo de realizar la evaluación psicológica del trastorno, identificado el padecimiento, es primordial evitar realizar el procedimiento quirúrgico y remitirlo a un profesional en salud mental.

El tratamiento de elección es la terapia cognitivo conductual; los inhibidores de la recaptura de serotonina pueden servir de ayuda tanto para la patología como para los trastornos de ansiedad que acompañan a la mayoría de los pacientes.

\section{DISCUSIÓN}

Navarro 0. señala que en la mayoría de los casos el TDC está sub diagnosticado, en un estudio de 17 pacientes con TDC, el trastorno solo se observó en 5 registros, y ninguno de los pacientes recibieron un diagnóstico oficial de TDC. Esta carencia de diagnósticos se debe a la reciente inclusión de la enfermedad en el DSM-IV; por lo tanto, el conocimiento clínico de la enfermedad, especialmente entre los médicos generales, no está muy extendido. La patología se asocia a menudo con conductas de vergüenza y secretismo, por lo tanto los pacientes a menudo no revelan sus inquietudes sobre su aspecto físico por temor a parecer superficiales o banales ante los médicos o familia.7 Las preocupaciones de estos pacientes con el TDC están relacionadas con el aspecto físico, una de las aparentes soluciones es ir donde el cirujano plástico. Sin embargo los sujetos no se 
benefician de la cirugía plástica, regresan para nuevas intervenciones y en algunos casos presentan demandas por negligencia. (Barlow/ Durand 2001).

Muchos de ellos después de recibir los tratamientos estéticos se sienten aún más feos y culpan al médico ya que su insatisfacción proviene de un malestar psicológico. No importa cuántas cirugías se hagan, este tipo de pacientes, siguen buscando defectos corporales, por lo que requieren terapia psicológica.

Estos pacientes tienen un alto índice de intentos o ideas suicidas. Según la revista americana de psiquiatría se examinaron a 185 sujetos durante 4 años con TDC. Los resultados mostraron que la idea suicida fue reportada en el $57.8 \%$ de los sujetos por año, y un promedio de $2.6 \%$ fueron intentos de suicidio también por año.

Muchos individuos con TDC no poseen conocimientos o comprensión de su enfermedad, y consideran su problema de naturaleza estética en vez de psicológica, por lo tanto, las personas que sufren de dismorfofobia buscan tratamiento en profesionales especialistas en medicina y cirugía cosmética, en lugar de tratamiento psicológico o psiquiátrico.8.

También se ha podido reconocer que una mejor conciencia de enfermedad por parte de los pacientes se asocia a un mejor pronóstico, así como identificar las partes del cuerpo más frecuentemente implicadas.

Todo paciente en el que se sospeche TDC debe ser derivado a un psiquiatra, siendo el tratamiento de elección psicoterapia y farmacoterapia El estrés que significa para estos pacientes el no ver una mejoría tras someterse a una cirugía puede empeorar la condición.

La agresión verbal, física e incluso las acciones judiciales pueden evitarse de esta forma si logramos excluir los casos de afectación grave. Por lo tanto se debe tener mucho criterio en el momento de seleccionar a los pacientes candidatos para someterse a una intervención de Cirugía Estética.

Es perentorio ampliar los estudios existentes sobre la materia, para poder caracterizar mejor la población consultante y diseñar estrategias rápidas de cribado, fáciles de implementar costo efectivas (6). Por ahora, se recomienda que el tema sea abordado en los cursos de formación para cirujanos plásticos.9.

\section{CONCLUSIONES}

El Cirujano Plástico debe tener mucho criterio en el momento de seleccionar a los pacientes que desean someterse a una intervención de Cirugía Estética.

Se recomienda el contacto cercano con especialistas psiquiatras calificados, de forma que en estos casos, sea posible tratar a los pacientes con TDC de forma multidisciplinaria.

La literatura señala la importancia de detectar a los pacientes con TDC, radica en ser considerada una enfermedad severa que tiende a la cronicidad, y en la mayoría de los casos no recibe tratamiento, lo cual convierte a este padecimiento en un problema, pese a que existe tratamiento farmacológico con inhibidores de la recaptura de serotonina, no todos los pacientes con trastorno dismórfico corporal (TDC) responden al tratamiento o reciben la atención psiquiátrica adecuada, por lo que muchos pacientes optan por tratamientos no psiquiátricos como lo es la cirugía plástica, que no resolverá el trastorno, corriendo el riesgo de afrontar los conflictos de estos pacientes, afrontar quejas y demandas infundadas e innecesarias.

\section{BIBLIOGRAFÍA}

1.-Barlow David, Suicidio y Trastorno Dismórfico corporal: Un estudio anticipado Psicología Anormal. American Psychiatric Publishing.2000. Vol. 5: 1, pp. 27-43

2.-Blackburn VF, Blackburn a V.: Taking a history in aesthetic surgery: SAGA--the surgeon's t ool for patient selection. J Plast Reconstr Aesthet Surg 2008; 61: 723-729.

3.-Borda T, Neziroglu F, Santos N, Donnelly K, RiveraRP.: Status of body dysmorphic disorder in Argentina. JAnxiety Disord 2011; 25: 507-512.

4.-Darryl James Hodgkinson, Identifying the Body-Dysmorphic Patient in Aesthetic Surgery, Double Bay, Australia .Aesth. Plast. Surg.2005.Rev. 29:503-509

5.-Gupta MA, Gupta AK.: Evaluation of cutaneous body image dissatisfaction in the dermatology patient. Clin Dermatol; 2013 Jan-Feb;31:1:72-79.

6.-Morselli PG, Boriani F.: Should plastic surgeons operateon patients diagnosed with body dysmorphic disorders? Plast Reconstr Surg 2012; 130: 620-622

7.-Navarro 0. Trastornos somatomorfos, Revista Mexicana de Neurociencia, 2009 Rev. 10:1:34-43

8.-Pavan C A P. Simonato. Psychopathologic Aspects of Body Dysmorphic Disorder:Aesth Plast Surg.2008 32:473-484.

9.-Rochefort-Ciscutti, G., The importance of diagnosing body dysmorphic disorder in the Aesthetic Surgery consultation. Cir. plást. iberolatinoam.- Julio - Agosto - Septiempbre 2014 Vol. 40 - № 3 Pag. 253-259. 